\title{
Queuing Game Theory Based Optimal Routing Scheme for Heterogeneous Users over Space Information Networks
}

\author{
Chao Guo, ${ }^{1}$ Cheng Gong, ${ }^{2}$ Haitao Xu, ${ }^{2}$ and Zhiyong Yao ${ }^{2}$ \\ ${ }^{1}$ Communication Engineering Department, Beijing Electronics Science and Technology Institute, Beijing 100070, China \\ ${ }^{2}$ School of Computer and Communication Engineering, University of Science and Technology Beijing, Beijing 100083, China \\ Correspondence should be addressed to Chao Guo; guo99chao@163.com
}

Received 26 July 2016; Revised 21 November 2016; Accepted 18 December 2016; Published 23 January 2017

Academic Editor: Vladimir Turetsky

Copyright (c) 2017 Chao Guo et al. This is an open access article distributed under the Creative Commons Attribution License, which permits unrestricted use, distribution, and reproduction in any medium, provided the original work is properly cited.

\begin{abstract}
An optimal routing scheme in space information networks was presented to balance network loads for heterogeneous users. According to the competition among the nodes, the model was built based on queuing game theory. The virtual routing platform was in charge of resources allocation and route selection. It got user's gain to decide which node the user joined in. Owning to the existing of heterogeneous users, an optimal admission fee needed to be obtained to avoid congestion. In our model, firstly, the whole welfare of the system was formulated. Then the optimal admission fee was calculated through maximizing the whole welfare. Meanwhile, the average maximum queue length was generated to set the buffer space of the node. At last, a routing factor was introduced into the route algorithm in order that the optimal routing could be selected by heterogeneous users. As a result, the system welfare reaches the maximum.
\end{abstract}

\section{Introduction}

In the space information networks, various types of users cause different service requirements and urgency levels. For example, aircrafts ask for high real-time service and disaster relief centers need to get priority for using communication resources. However, this is hard to accomplish due to the network characteristics of the long delay and high link failure rate [1]. In most cases, the users are treated as one or limited few types to reduce the network resource consumption $[2,3]$. These schemes simplify the system model, but ignore the real situation of space information networks. In order to improve the network performance, congestion control should be paid more attention [4]. Users with different service requirements and urgency are called heterogeneous users. They join a queue of a node depending on their own information. In contrast, users with lack of distinction accept nontargeted services. Obviously, it is unfavorable for the rational allocation of limited resources and congestion avoidance. The purpose of each user is to achieve the transmission of information under the premise of satisfying their demands. Therefore, a suitable or no-routing algorithm is particularly important.
In this paper, we build a system model of space information networks for heterogeneous users and then formulate this model using queuing game theory [5]. At last, an optimal routing based queuing game theory for heterogeneous users over space information networks (RQGH) algorithm is proposed to improve the network performance. We classify users by their different benefit value which will be obtained after completing service. It is assumed that the user's benefit is a random variable with continuous distribution. The more urgent is the larger the value of user's benefit is. The reason is the randomness and unexpected of users in space information networks. A user announced its benefit to the accessible nodes, and then the nodes which are the players of our game model choose the strategies according to their situation. On the basis of this process, users get the corresponding suitable service. High emergency user can be serviced when the normal user balks. It takes the heterogeneous users into account under the situation of overall fairness. Utilizing the optimal result of the built system model, we design the RQGH algorithm. It introduces a routing factor which is composed of the optimal admission fee, the current waiting cost, and the routing transmission cost. The first two parameters describe 
the state of the current routing node. The third parameter indicates the work situation of the whole route.

The rest of this paper is organized as follows. Section 2 introduces some related works. In Section 3, the theoretical framework is described through the system architecture and resource optimization of the routing node. Our proposed RQGH algorithm is given in Section 4. Simulations and comparisons are done in Section 5. At last, a conclusion is drawn in Section 6.

\section{Related Work}

Since satellites were sent into space, the researches about routing and resource allocation have been conducting. How to promote the use efficiency of satellite resources is a hot topic because of the high costs of space communication. Some of outcomes are shown in $[6,7]$ to solve the problem from the perspective of network topology. In recent years, many routing schemes using game theory and optimization theory have appeared in the literatures [8-10]. As a result, the overall state of the network can be optimized on the theoretical level.

In [6], the authors designed a predictable mobile-routing protocol by developing a dynamic topology model for spacecraft networks. The predictable and unpredictable changes of networks were described in this topology model which is based on a notion of snapshots. Then they presented a topology information management algorithm to support the predictable link-state-based routing scheme. Therefore, the proposed routing protocol was able to handle both predictable and unpredictable changes, as well as their interaction.

In [7], a virtual topology model was clarified based on Virtual Node method for low earth orbit satellite networks. It formalized the definition and designed routing protocol to reduce the requisite snapshots, optimize the snapshot length, and prevent path stretch and contraction. Furthermore, the deficiency of the satellite group manager method could be overcome in multilayered satellite networks.

In [8], the authors used the differential game theory to formulate a dynamic power allocation model. Then they solved it and obtained a feedback Nash equilibrium solution for transmitted power. Based on this model, a joint cross-layer optimized routing was proposed in deep space information networks. They introduced a concept of the hybrid link homeostasis in order to describe the routing metric. This polynomial time algorithm could choose routing joint, allocate the transmission power, and form a predictable contact schedule at the same time. The effectiveness and feasibility were demonstrated by the numerical results.

In [9], the authors controlled the admission price to determine whether customers join the queue for service in a multiple traffic environment. Load balancing in routing games was taken into account for $M / M / 1$ type servers. One of their considered schemes was that each customer selects a server according to the optimal probability. The other was that some of the customers chose a server with probabilities of minimized total cost, and others adopted the first scheme.
In [10], the authors utilized dynamical reference queue length to overcome the difficulty for satellite terminals to get unavailable information based on game theory. They focused on the scheme of queue length for enhancing the effectiveness of bandwidth allocation in broadband satellite networks. Through using the congestion pricing theory, a simple and effective Bandwidth-on-Demand algorithm was proposed with significant network information.

This paper researches some new points following our previous work [11, 12]. In [11], a preliminary queue congestion control model was presented on the basis of queuing game theory. It focused on the situation of leaving impatient users in space information networks. However, the different service requirements were not considered in this scheme. In [12], service scheduling in Cloud Computing was studied based on queuing game theory. Through controlling the service scheduling strategy, this scheme maximized the Cloud Computing platform's total payoff. However, the model only considered that when a user's service request came, it would decide whether to join or balk the queue according to the value of the user's gain.

All the above researches explored solution schemes of routing problems or resource allocation, although adopting different methods in various environments. Comparing with their solutions, our work is focused on distinguishing heterogeneous users with various service requirements and emergency degree in space information networks. An effective routing scheme is then designed after the procedure of searching the optimal admission fee.

\section{Theoretical Frameworks}

We build a virtual routing platform (VRP) to discuss the resource optimization for a single node and then formulate the routing issue for the space information networks with multiple nodes. Considering the heterogeneous users, the arrival user's benefit is supposed to a random variable based on the queuing game theory. The buffer length of each node is finite. For single node in VRP, a user decides whether to join the buffer queue depending on its gain. The overall welfare consists of the user's gain and the node's profit. We can obtain an optimal condition after formulating and analyzing the overall welfare. Under the situation of multiple nodes in VRP, the optimal condition is utilized to construct a routing factor. The user chooses a path with the lowest routing factor to transmit information and achieve the optimal routing at last.

3.1. Architecture of the System. Figure 1 describes a routing model which contains multiple nodes using the RQGH algorithm. The VRP concludes $m$ nodes of the space information networks, $m=1,2, \ldots, j\left(j \in N^{+}\right)$. Each node has a route buffer for providing users the queue space to wait for forwarding. Unlike the model in [13], the route buffer in our model is finite with a buffer length $n$. Therefore, we formulate an $M / M / 1 / n$ queuing game model for resource optimization of a single node. In our model, heterogeneous users enter the VRP and ask for routing services. The heterogeneous 


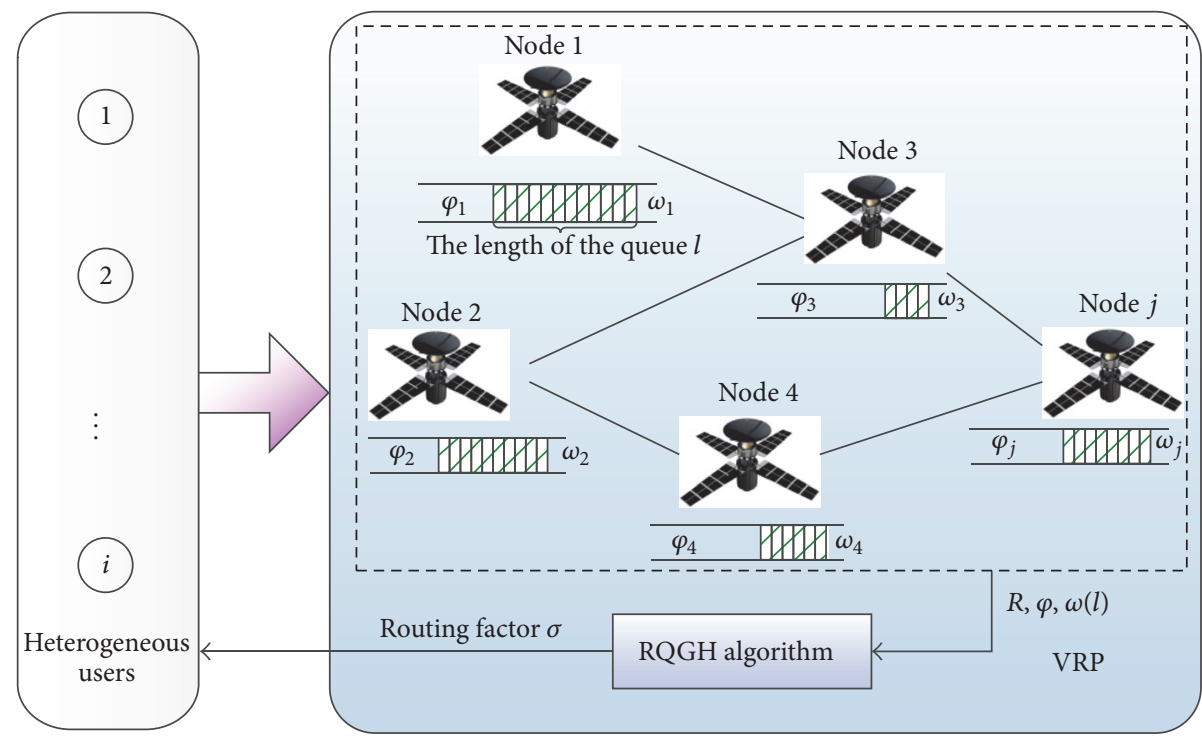

FIGURE 1: A multiple nodes routing model using the RQGH algorithm.

characteristics of the users are denoted by the random variation of the users' benefit with a distribution function $\Psi$ as shown in Definition 1. For multiple nodes in the VRP, the nodes calculate their user's gain $G(l)$ and allow the user to enter the node $j$ which has the maximum $G_{j}(l) . l$ is the queue length and $0 \leq l \leq n\left(l \in N^{+}\right)$. When the nodes are full, they will refuse to provide the service for any user. Therefore the game occurs under the condition $0 \leq l<n$. Based on the result of resource optimization for a single node, all the nodes in the multiple nodes model can calculate their routing factors $\beta$ which consist of the current costs and the routing transmission costs. When a user enters the VRP, the corresponding routing factor of each alternative node is announced to the VRP, and then the VRP chooses the node with the lowest routing factor to allow the user being serviced. It achieves an optimal routing. Note that a node may obtain more than one routing factor due to the multiple routing choices.

Definition 1. For multiple nodes in VRP, a user pays an admission fee $\varphi_{j}$ to the node $j$ for getting routing service. Its benefits which are obtained after being serviced completely by the node are a random variable $R$ with a distribution function $\Psi$. We assume that it is a normal distribution. The mean and the variance of distribution function $\Psi$ are assumed as $\varepsilon$ and $\sigma^{2}$, respectively. The user decides to join the queue of this node $j$ only if its gain $G_{j}(l)$ is the maximum.

From this definition, the heterogeneous users' benefits $R$ are different in the space information networks. A stationary Poisson stream of the user arrives to a VRP with parameter $\lambda$. The service times of the routing nodes in the space information network are independent, identically, and exponentially distributed with parameter $\mu$. The system's utilization factor is defined as $\rho=\lambda / \mu$ [5]. For stability, we assume that $0<$ $\lambda<\mu$. According to the user's benefit and the waiting cost, we can calculate the user's gain $G_{j}(l)$. If $G_{j}(l)$ is the maximum, the user joins the queue. Then the whole welfare $W_{j}(\varphi)$ is obtained. In order to get the optimal $W_{j}(\varphi)$, we need to find the most suitable admission fee $\varphi_{j}$.

Definition 2. In the routing model with multiple nodes, the nodes announce routing factors $\beta$ to the VRP after a user entrance. It contains the following three parts. The first is the optimal admission fee $\varphi^{*}$ of each node. The second is the current waiting cost $\omega(l)$ which is relevant to the number of users in the queue. The last is the routing transmission cost $D(\theta)$ which is determined mainly by the transmission distance and the link failure rate. $\theta$ denotes the routing transmission cost per unit of distance.

From this definition, we obtain the optimal admission fee $\varphi^{*}$ of each node by resource optimization and utilize it as a component part of the routing factor. It is of benefit for guaranteeing the individual optimal of each routing node. By such analogy, the routing factor is defined to make sure that the optimal routing can be selected.

Specifications of the RQGH algorithm parameters are given in the following part.

Specifications of the Parameters of RQGH Scheme for Single Node

$\lambda$ : Arrival rate of user in a VRP

$\mu$ : Service rate of the node in a VRP

$\rho$ : Utilization factor $\rho=\lambda / \mu$

$l$ : Queue lengths in a node, $l \in N^{+}$

$\varphi$ : An admission fee $\varphi$

$R$ : A user's benefit from completing service which is a random variable

$C$ : The cost to a user of staying in the system per unit of time 


\section{$\beta$ : A routing factor}

$\theta$ : The routing transmission cost per unit of distance $q_{l}$ : The probability of observing $l$ users in the queue.

3.2. Resource Optimization of the Routing Node. In order to optimize routing selection of the whole space information networks, we first should make each node resource optimization. A user pays an admission fee $\varphi_{j}$ to the node $j$ before getting routing service and receives a benefit $R$ once to be serviced completely. In this process, the user also needs to pay for waiting in the queue. The charge $\omega_{j}(l)$ depends on the queue length. Therefore, the user's gain is obtained as $G_{j}(l)=R-\varphi_{j}-\omega_{j}(l)$. If the user's gain is the maximum, it will be selected to the node. As we discussed above, the user's benefit is a random variable with distribution function $\Psi$. Given a maximum queue length of $n$, the probability of observing $l$ users in the system is $p_{l}$. The node's profit is

$$
Z\left(\varphi_{j}\right)=\lambda\left[1-\Psi\left(\varphi_{j}+\omega_{j}(l)\right)\right]\left(1-p_{n}\right) \varphi_{j},
$$

where $\omega_{j}(l)=C((l+1) / \mu)$. $\left[1-\Psi\left(\varphi_{j}+\omega_{j}(l)\right)\right]$ denotes the probability of a user joining the queue of the node.

The whole welfare $W(\varphi)$ of the system can be specified as

$$
\begin{gathered}
W\left(\varphi_{j}\right)=\lambda \sum_{l=0}^{n} p_{l}\left[1-\Psi\left(\varphi_{j}+\omega_{j}(l)\right)\right] \\
\cdot\left[E\left(R \mid R>\varphi_{j}+\omega_{j}(l)\right)-\omega_{j}(l)\right],
\end{gathered}
$$

where $\left[E\left(R \mid R>\varphi_{j}+\omega_{j}(l)\right)-\omega_{j}(l)\right]$ denotes the sum of the user's gain and the node's profit when the queue length is $l$.

For the imposed admission fee $\varphi$, the generated impact for each node in the system is the same if the users are the same. However, in the space information networks, more and more heterogeneous users need to be serviced. Selected admission fee affects the system performance and the service fairness. Based on queuing game theory, we formulate a resource optimization model for a single node in the VRP. The queue model is $M / M / 1 / n$.

Theorem 3. For a single node resource optimization model, the heterogeneous users in the space information networks are chosen by the node $j$ according to their respective benefits. Under the same state of the queue, the admission fee determines the user's gain which indicates whether to join the queue or not. In order to maximize the whole welfare, the optimal admission fee is

$$
\begin{gathered}
\varphi_{j}^{*}=\underset{\varphi_{j}}{\arg \max }\left\{\lambda \sum_{l=0}^{\infty} p_{l}\left[\int_{\varphi_{j}+\omega_{j}(l)}^{+\infty} \frac{t}{\sqrt{2 \pi}} e^{-(t-\varepsilon)^{2} / 2} d t-\omega_{j}(l)\right]\right. \\
\left.\cdot \int_{\varphi_{j}+\omega_{j}(l)}^{+\infty} \frac{1}{\sqrt{2 \pi}} e^{-(t-\varepsilon)^{2} / 2} d t\right\} .
\end{gathered}
$$

Proof. The distribution function of user's benefit $R$ can be formulated as

$$
\Psi(x)=\int_{-\infty}^{x} \frac{1}{\sqrt{2 \pi} \sigma} e^{-(t-\varepsilon)^{2} / 2 \sigma^{2}} d t,
$$

where $\Psi(x)$ equals the probability of $R \leq x$ which is denoted as $P\{R \leq x\}$.

While the system is in the state $l$, the expected whole welfare gained from a user who arrives is

$$
\begin{aligned}
W_{l}(\varphi)= & {\left[E\left(R \mid R>\varphi_{j}+\omega_{j}(l)\right)-\omega_{j}(l)\right] } \\
& \cdot \int_{\varphi_{j}+\omega_{j}(l)}^{+\infty} \frac{1}{\sqrt{2 \pi} \sigma} e^{-(t-\varepsilon)^{2} / 2 \sigma^{2}} d t,
\end{aligned}
$$

where $\left[E\left(R \quad \mid \quad R>\varphi_{j}+\omega_{j}(l)\right)-\omega_{j}(l)\right]=$ $\left[\int_{\varphi_{j}+\omega_{j}(l)}^{+\infty}(t / \sqrt{2 \pi} \sigma) e^{-(t-\varepsilon)^{2} / 2 \sigma^{2}} d t-\omega_{j}(l)\right]$.

With the restriction of the buffer length $n$, the average welfare contribution of the system per unit of time is

$$
W\left(\varphi_{j}\right)=\lambda \sum_{l=0}^{n} p_{l} W_{l}\left(\varphi_{j}\right)
$$

In order to calculate the optimal admission fee, we first solve the welfare with $l \in[0,+\infty)$. Under the stable statement [14], we can get the following algebraic equations.

For 0 state, $\lambda_{0} p_{0}=\mu p_{1}$ such that $p_{1}=\left(\lambda_{0} / \mu\right) p_{0}$.

For 1 state, $\lambda_{1} p_{1}=\mu p_{2}$ such that $p_{2}=\left(\lambda_{1} \lambda_{0} / \mu^{2}\right) p_{0}$. ...

For $l-1$ state, $\lambda_{l-1} p_{l-1}=\mu p_{l}$ such that $p_{l}=\left(\left(\lambda_{l-1} \cdot \ldots \cdot\right.\right.$ $\left.\left.\lambda_{1} \lambda_{0}\right) / \mu^{l}\right) p_{0}$.

When $l$ users are present, the joining arrival rate is

$$
\lambda_{l}=\lambda \int_{\varphi_{j}+\omega_{j}(l)}^{+\infty} \frac{1}{\sqrt{2 \pi}} e^{-(t-\varepsilon)^{2} / 2} d t
$$

Because of the regularity condition $\sum_{l=0}^{\infty} p_{l}=1, p_{0}$ can be calculated as

$$
p_{0}=\left(1+\sum_{l=0}^{\infty} \rho^{l+1} \prod_{i=0}^{l} \int_{\varphi_{j}+\omega_{j}(i)}^{+\infty} \frac{1}{\sqrt{2 \pi}} e^{-(t-\varepsilon)^{2} / 2} d t\right)^{-1} .
$$

Then the whole welfare $W(\varphi)$ can be rewritten as

$$
\begin{gathered}
W\left(\varphi_{j}\right)=\lambda \sum_{l=0}^{\infty} p_{l}\left[\int_{\varphi_{j}+\omega_{j}(l)}^{+\infty} \frac{t}{\sqrt{2 \pi}} e^{-(t-\varepsilon)^{2} / 2} d t-\omega_{j}(l)\right] \\
\cdot \int_{\varphi_{j}+\omega_{j}(l)}^{+\infty} \frac{1}{\sqrt{2 \pi}} e^{-(t-\varepsilon)^{2} / 2} d t .
\end{gathered}
$$

After optimizing the formula (9), we can obtain the optimal admission fee as

$$
\begin{aligned}
\varphi_{j}^{*} & =\underset{\varphi_{j}}{\arg \max }\left\{\lambda \sum _ { l = 0 } ^ { \infty } p _ { l } \left[\int_{\varphi_{j}+\omega_{j}(l)}^{+\infty} \frac{t}{\sqrt{2 \pi}} e^{-(t-\varepsilon)^{2} / 2} d t\right.\right. \\
& \left.\left.-\omega_{j}(l)\right] \int_{\varphi_{j}+\omega_{j}(l)}^{+\infty} \frac{1}{\sqrt{2 \pi}} e^{-(t-\varepsilon)^{2} / 2} d t\right\} .
\end{aligned}
$$

Theorem 4. For a single node resource optimization model, to make sure that the most heterogeneous users in the space 
information networks should be serviced in our model, the constant $\alpha$ is introduced. When $G(l) \geq \alpha$, most users will be serviced. Therefore, the average maximum queue length $l_{\max }$ with this restraint condition is

$$
l_{\max }=\left\lfloor\mu \frac{\varepsilon-\varphi_{j}^{*}-\alpha}{C}-1\right\rfloor,
$$

where $\varepsilon$ denotes the expectation of a random variable $R$.

Proof. According to Theorem 3, the ith user's gain can be rewritten as

$$
G_{i j}(l)=R_{i}-\varphi_{j}^{*}-\omega_{j}(l),
$$

where $R_{i}$ denotes $i$ th user's benefit.

Only when $G(l) \geq \alpha$, most users will be serviced. Then we can get

$$
G_{i j}(l) \geq \alpha
$$

Taking into account the overall situation of the model, we utilize the expectation of a random variable $R$ to satisfy node resource allocation in most cases. A restraint condition of the queue length $l$ is

$$
\varepsilon-\varphi_{j}^{*}-C \frac{l+1}{\mu} \geq \alpha .
$$

Then we can obtain the restraint condition of queue length as follows:

$$
l \leq \mu \frac{\varepsilon-\varphi_{j}^{*}-\alpha}{C}-1 .
$$

Because the queue length is an integer, we can get the maximum queue length as

$$
l_{\max }=\left\lfloor\mu \frac{\varepsilon-\varphi_{j}^{*}-\alpha}{C}-1\right\rfloor .
$$

In the space information networks, our queuing game model is $M / M / 1 / n$. With the restraint condition of formula (11), the value of $n$ needs to be set more than $l_{\max }$ so as to obtain the whole optimal welfare $W\left(\varphi_{j}^{*}\right)$. With the consideration of the real network environment, we spread the above results to our multiple nodes routing model. Resource optimization flow of the routing nodes is illustrated in Figure 2. Firstly, we calculate the whole welfare of each node in VRP. Then an optimal admission fee of the corresponding node is obtained by maximizing the whole welfare. After that, we evaluate the user's gain using the average of the user's benefit $R$ to get the maximum queue length of each node. It is useful for defining the buffer length of nodes. When new users arrive, the VRP announces which node is selected according to the optimal admission fee and the current queue length of nodes. If all nodes are full, users will balk and wait for retransmission. Else the user will be selected by the node with the maximum value of the gain.

\section{An Optimal Routing Algorithm Based on Queuing Game Theory}

In space information networks, various nodes provide service for heterogeneous users. To make sure that the optimal routing can be selected to users, we design a RQGH algorithm. The most important part of this algorithm is a routing factor $\beta$ of each node. As Definition 2, it consists of the optimal admission fee $\varphi^{*}$, the current waiting cost $\omega(l)$, and the routing transmission cost $D(\theta)$. When heterogeneous users enter the VRP, the nodes announce the users' gain and the top one should be selected for users to get routing service. This selected node checks its routing table and chooses the route with minimum routing factor. Routing factor $\beta$ of the node $j$ is calculated as

$$
\beta_{j}=\sum \varphi^{*}+\omega(l)+D(\theta),
$$

where $D(\theta)=\theta d ; d$ denotes the information transmission distance between the current node and the destination node.

According to the above discussion, we know that the optimal admission fee $\varphi^{*}$ is a constant for the same nodes. In the whole routing system, $\sum \varphi^{*}$ describes the influence of the number of hops. The routing distance $D(\theta)$ also can be sure after selecting a route and the delay status can be considered by it. However, the current waiting cost is dynamic changing with the changing number of users in the queue. $\omega(l)$ expresses the current situation of the routing nodes.

The RQGH algorithm is designed based on the result of a queuing game model and takes the hops and the information transmission distance of routing into account. So an optimal routing can be obtained to allocate the resources reasonability and improve the efficiency of the network. When users enter the VPR, they focus on the situation of their gain from all the available nodes. If the user's gain is the maximum, the corresponding node is one of the eligible service nodes. The user is selected to the node which generates the maximum gain and joins the queue. Then this node is considered as a sending node. We check its routing table for accessible routes and calculate the routing factors of each path. After comparing the value of the routing factors, the route with minimum routing factor is determined as the user's path. While the user joins the queue of the destination node, the ACK is returned to the sending node, as shown in Algorithm 1.

\section{Simulations and Comparisons}

In this section, we concentrate on the performance simulation of the proposed RQGH algorithm in a VRP of space information networks. We will give two simulations to demonstrate the efficiency of our proposed model. In the first numerical simulation, the optimal admission fee is evaluated under the determinate network environment. Then the performance of our scheme will be shown through changing the value of system parameters. In the second system simulation, we will compare the end-to-end delay and 


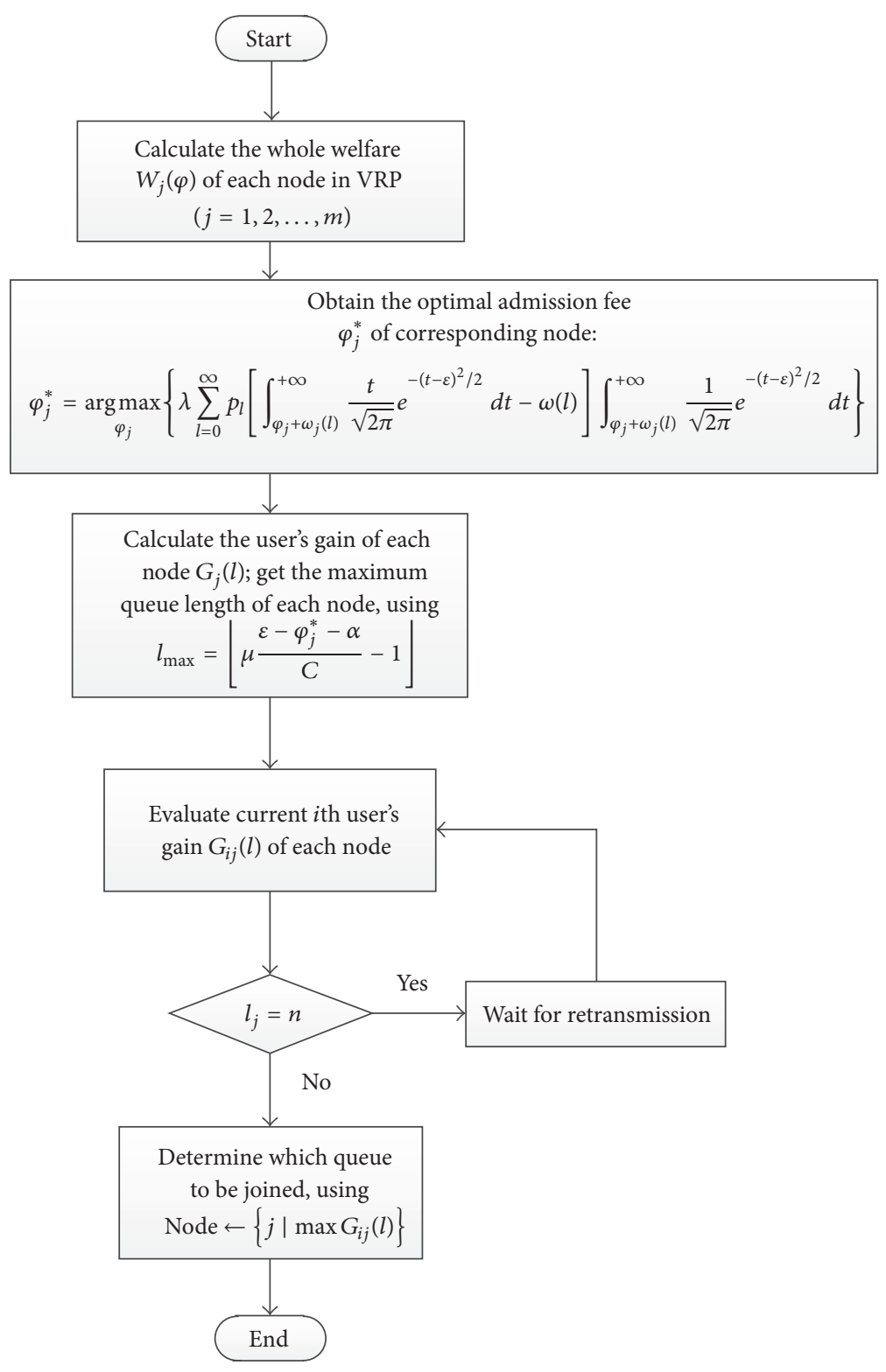

FIGURE 2: Resource optimization flow of the routing nodes.

the throughput of the traditional algorithm and our RQGH algorithm.

In the first numerical simulation, the experiments and analysis are divided into the following three steps. Firstly, we get the node's profit and the whole welfare with different admission fees according to our system model. The admission fee which corresponds with the peak value of the whole welfare is the optimal admission fee $\varphi^{*}$. Secondly, the trends of the whole welfare and the optimal fee are described under different levels of congestion. At last, the average maximum queue length is calculated with the corresponding optimal admission fee. The value of the common simulation parameters is defined as in Table 1.

We define $R \in[80,120]$ to simplify the following calculation. The node's profit and the whole welfare with different admission fees are presented in Figure 3. The whole welfare
TABLE 1: The value of the common simulation parameters.

\begin{tabular}{ccccc}
\hline$\lambda$ & $\mu$ & $C$ & $\varepsilon$ & $\sigma$ \\
\hline 50 & 60 & 10 & 100 & 20
\end{tabular}

consists of the node's profit and the user's gain. The intersection point of the solid curve and the dotted curve means that the user's gain equals zero at the corresponding admission fee. With the increase of the admission fee, the whole welfare first increases and then decreases. In order to maximize the whole welfare, we obtain the optimal admission fee $\varphi^{*}$ denoted by the asterisk of the peak value of the solid curve.

In order to show the influence of congestion level of network performance, we simulate the whole welfare with the growing arrival rate. The parameter of the arrival rate is given 


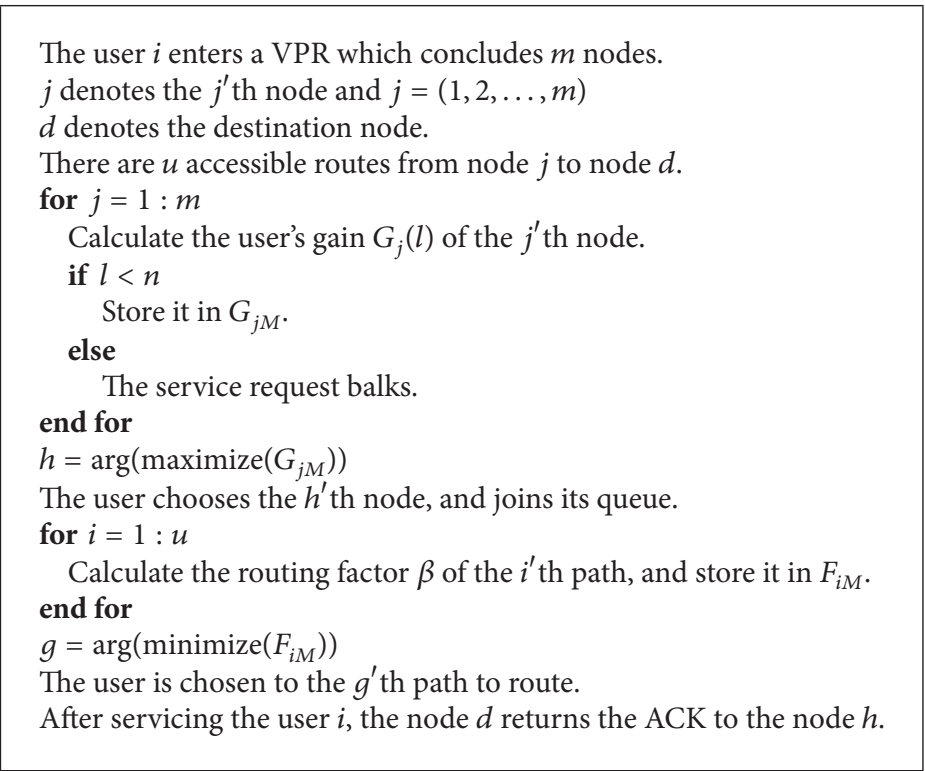

Algorithm 1: A routing algorithm based on queuing game theory for heterogeneous users.

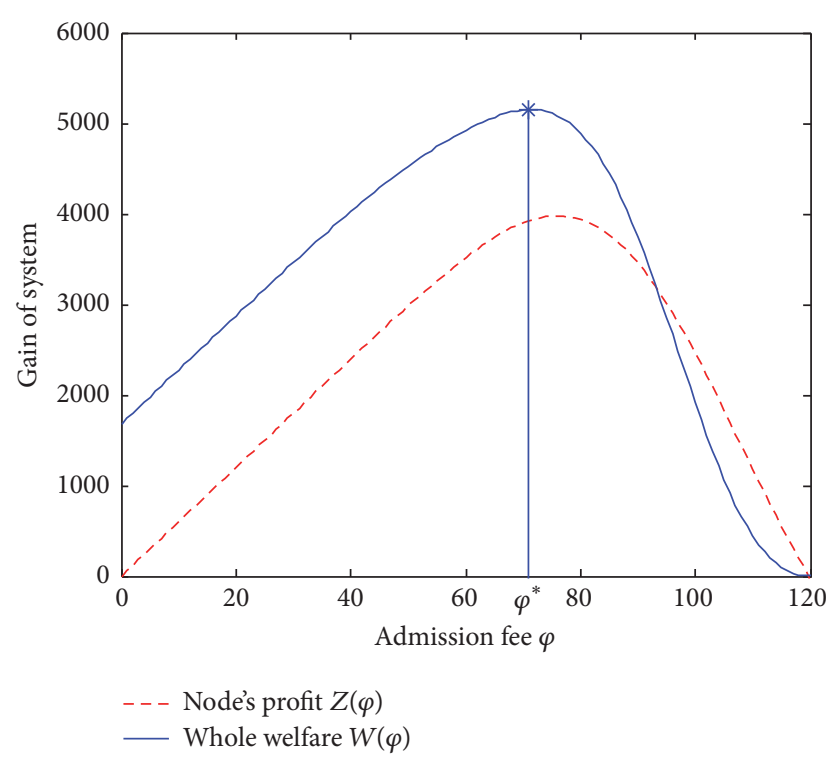

FIGURE 3: The node's profit and the whole welfare with different admission fees.

by $\lambda=\{50,53,56,59\}$ and the service rate remains unchanged. As shown in Figure 4, the larger the $\lambda$, the greater the maximum of the whole welfare. It means that our model is able to improve the upper bound of the network performance while the degree of congestion increases. Since the number of arriving users rises, the system balances the load through increasing the optimal admission fee. As a consequence, we observe that the corresponding optimal admission fee becomes bigger.

Due to the finite buffer of the node, the reasonable maximum queue length needs to be set. With the assumption $\alpha=$ 0 , Figure 5 gives the average maximum queue length $l_{\max }$ with

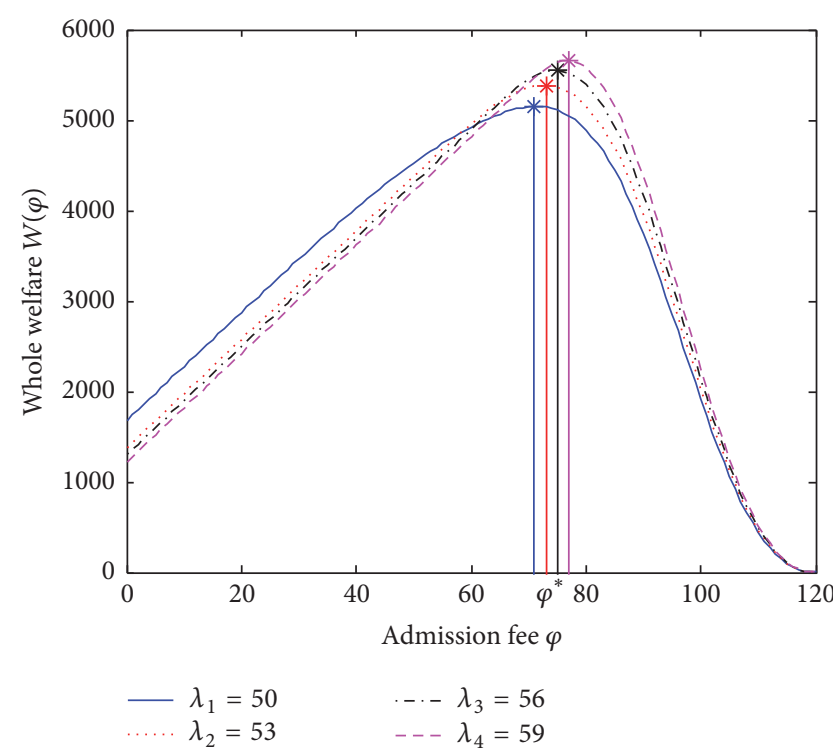

FIgURE 4: The comparison of the optimal admission fee for different arrival rates.

different optimal admission fees. Therefore, the buffer length of the node must be no less than $l_{\max }$ such that the system can achieve an optimal state. With the increase of the optimal admission fee, the average maximum queue length decreases. The cause is that the user's gain becomes smaller.

In the second realistic simulation, we compare the average end-to-end delay and the throughput of the route delivery rate of the traditional algorithm and our RQGH algorithm to show the improvement of the network performance. We select the DARTING which is proposed in [15] to simulate the traditional congestion control scheme. A realistic simulation environment is built by using the Opnet ${ }^{\circledR}[16]$. All the 


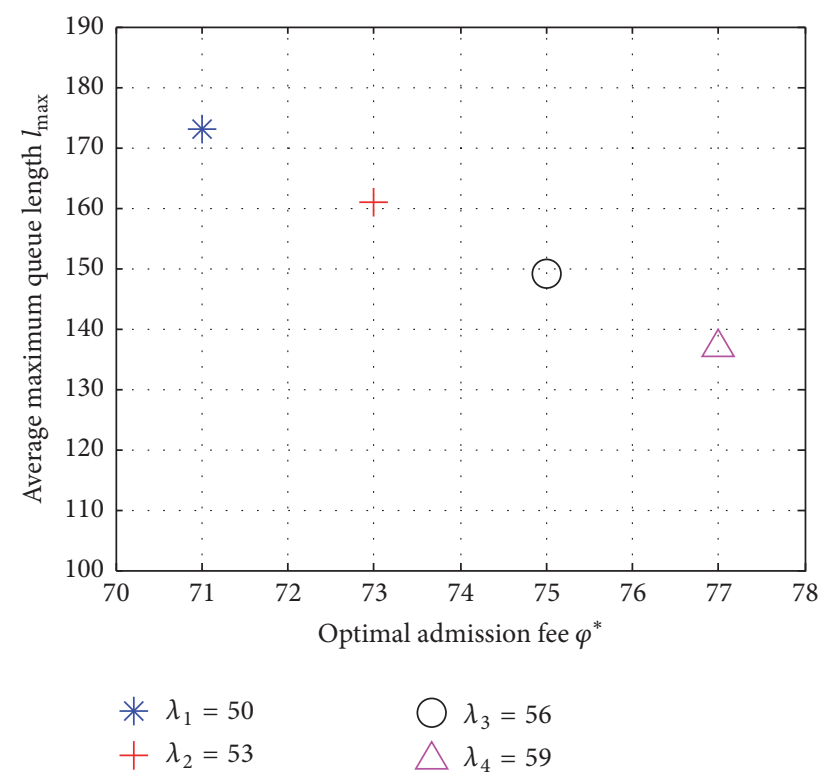

FIGURE 5: The average maximum queue length with different optimal admission fees.

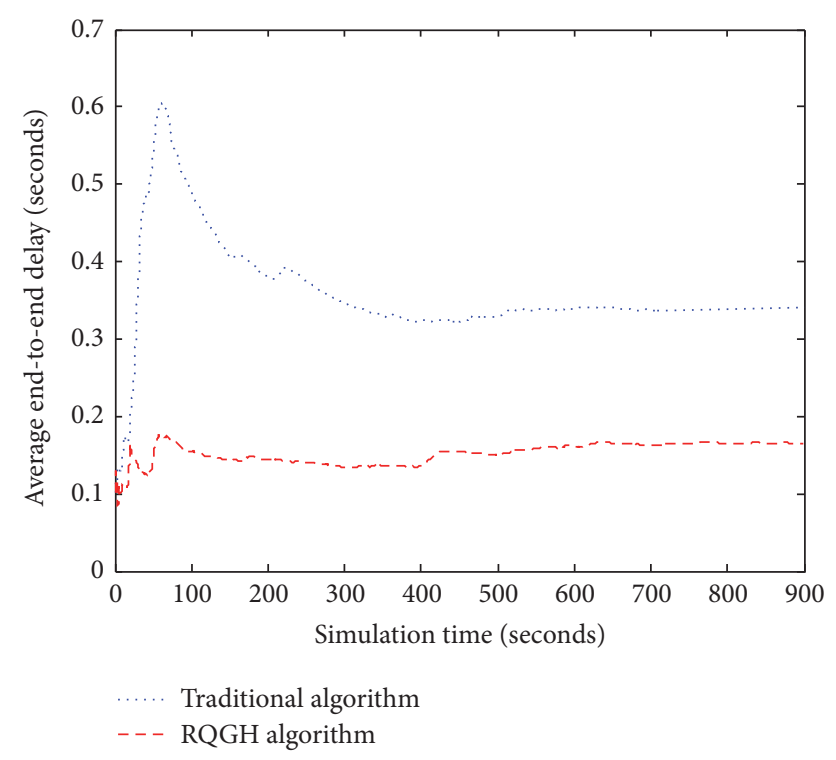

FIGURE 6: The comparison of average end-to-end delay between two algorithms.

schemes and data sources are simulated on the Intel Pentium ${ }^{\circledR}$ Dual-Core $3.00 \mathrm{GHz}$ using $\mathrm{C}$ language.

As shown in Figure 6, the average end-to-end delay of the RQGH algorithm is lower. The reason is that users are serviced according to their requirements. While in traditional algorithm, users with different emergency degree are treated the same. It saves waiting time and then improves the transmission efficiency.

From Figure 7, we can see that our proposed algorithm have the bigger throughput. The explanation is that the

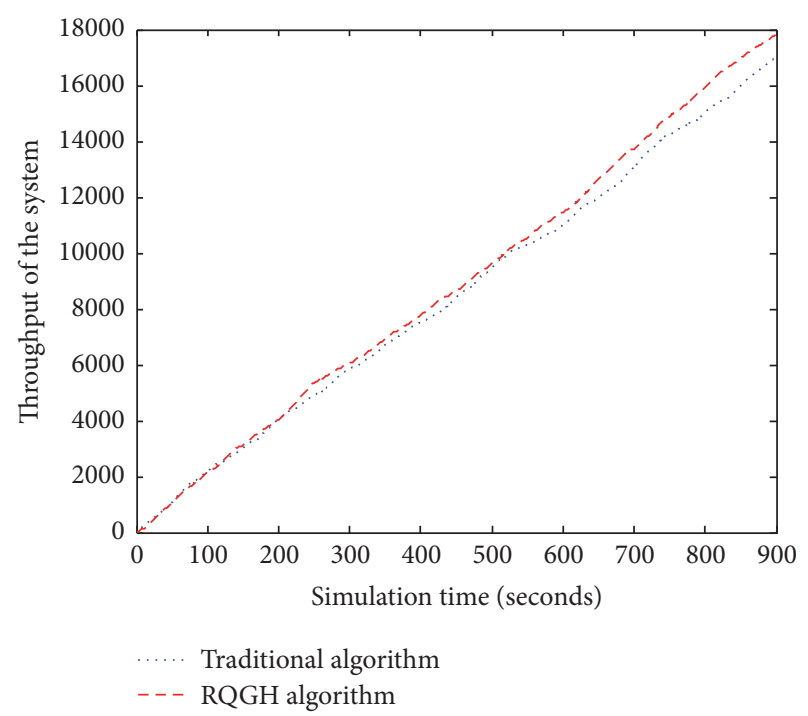

FIgURE 7: The comparison of throughput of the system between two algorithms.

designed RQGH algorithm utilizes the optimal admission fee as one part of the routing factor. It can improve the effectiveness of the system.

\section{Conclusion}

In space information networks, there are heterogeneous users due to different service requirements and urgency levels. RQGH scheme is proposed to achieve the optimal routing for heterogeneous users. It models the whole welfare of the system through the queuing game theory and calculates the optimal admission fee to make sure that the network performance achieves theoretical optimal state. According to the restriction of user's gain being no less than a constant, the average maximum queue length is evaluated with the corresponding optimal admission fee. Therefore, the buffer length of the node must be no less than the average maximum queue length to attain the maximum performance of the system. After a routing factor is introduced into our RQGH algorithm, users can select an optimal routing in space information networks. Because the routing factor consists of the optimal admission fee, the current waiting cost, and the routing transmission cost, it covers the current optimal state of the node and the routing cost situation. At the same time, the lowest routing factor implies an optimal routing among all accessible paths. On the other hand, the congestion problems are solved at a certain degree. The results of simulations show the optimal admission fee of the node and prove the feasibility of RQGH algorithm.

For the future work, we attempt to distinguish users by their priority levels which need to be bought. Priority level of the user is more practical for the limited network resources. Then how to select prices that induce users to buy the surefire priority level is the key. Finally, the whole welfare is maximized based on this solution. 


\section{Competing Interests}

The authors declare that there is no conflict of interests regarding the publication of this paper.

\section{Acknowledgments}

This work is supported by Fundamental Research Funds for the Central Universities of China and Ph.D. Start-Up Fund of Beijing Electronic and Technology Institute.

\section{References}

[1] C. Caini and R. Firrincieli, "Application of contact graph routing to LEO satellite DTN communications," in Proceedings of the IEEE International Conference on Communications (ICC '12), pp. 3301-3305, Ottawa, Canada, June 2012.

[2] H. Cruz-Sanchez, G. S. Staples, R. Schott, and Y.-Q. Song, "Operator calculus approach to minimal paths: precomputed routing in a store and forward satellite constellation," in Proceedings of the IEEE Global Communications Conference (GLOBECOM '12), pp. 3431-3436, IEEE, Anaheim, Calif, USA, December 2012.

[3] K.-C. Go, J.-H. Kim, J.-R. Cha, B.-G. Jo, and K.-K. Kim, "Challenges and solutions for routing in converged satellite and terrestrial networks," in Proceedings of the IEEE Military Communications Conference (MILCOM '13), pp. 1838-1843, San Diego, Calif, USA, November 2013.

[4] V. Ramaswamy, D. Choudhury, and S. Shakkottai, "Which protocol? Mutual interaction of heterogeneous congestion controllers," IEEE/ACM Transactions on Networking, vol. 22, no. 2, pp. 457-469, 2014.

[5] R. Hassin and M. Haviv, To queue or not to queue: equilibrium behavior in queueing systems, vol. 59 of International Series in Operations Research \& Management Science, Kluwer Academic Publishers, 2003.

[6] D. Fischer, D. Basin, K. Eckstein, and T. Engel, "Predictable mobile routing for spacecraft networks," IEEE Transactions on Mobile Computing, vol. 12, no. 6, pp. 1174-1187, 2013.

[7] Y. Lu, F. Sun, and Y. Zhao, "Virtual topology for LEO satellite networks based on earth-fixed footprint mode," IEEE Communications Letters, vol. 17, no. 2, pp. 357-360, 2013.

[8] L. Zhang and X. Zhou, "Joint cross-layer optimised routing and dynamic power allocation in deep space information networks under predictable contacts," IET Communications, vol. 7, no. 5, pp. 417-429, 2013.

[9] T. Bodas, A. Ganesh, and D. Manjunath, "Load balancing and routing games with admission price," in Proceedings of the 50th IEEE Conference on Decision and Control and European Control Conference (CDC-ECC '11), pp. 249-254, December 2011.

[10] A. Fiaschetti, A. Pietrabissa, M. Fiaschetti, and M. Petrone, "Congestion pricing for dynamic bandwidth allocation in satellite networks: a game-theoretic approach," in Proceedings of the IEEE 1st AESS European Conference on Satellite Telecommunications (ESTEL '12), pp. 1-6, IEEE, Rome, Italy, October 2012.

[11] C. Guo, X. Zhou, F. Lin, and S. A. M. Kasaei, "A congestion control model based on queuing game with impatient users for space information networks," Journal of Information and Computational Science, vol. 12, no. 8, pp. 3319-3331, 2015.

[12] F. Lin, X. Zhou, D. Huang, W. Song, and D. Han, "Service scheduling in cloud computing based on queuing game model,"
KSII Transactions on Internet and Information Systems, vol. 8, no. 5, pp. 1554-1566, 2014.

[13] C. Larsen, "Investigating sensitivity and the impact of information on pricing decisions in an $\mathrm{M} / \mathrm{M} / 1 / \infty$ queueing model," International Journal of Production Economics, vol. 56-57, pp. 365-377, 1998.

[14] W. Usaha and J. Barria, "Markov decision theory framework for resource allocation in LEO satellite constellations," IEE Proceedings: Communications, vol. 149, no. 5-6, pp. 270-276, 2002.

[15] K. Tsai and R. P. Ma, "Darting: a cost-effective routing alternative for large space-based dynamic-topology networks," in Proceedings of the Military Communications Conference (MILCOM '95), pp. 682-686, San Diego, Calif, USA, November 1995.

[16] Tool by Opnet ${ }^{\circledR}$ Technologies-http://www.opnet.com. 


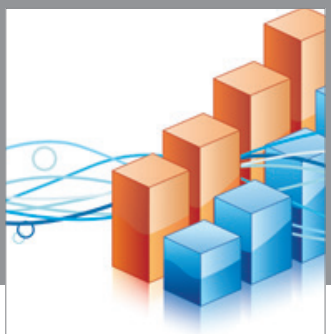

Advances in

Operations Research

vatem alat4

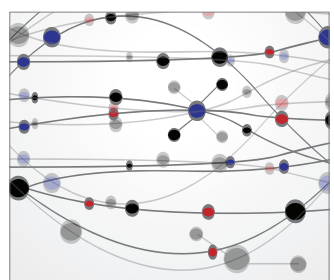

\section{The Scientific} World Journal
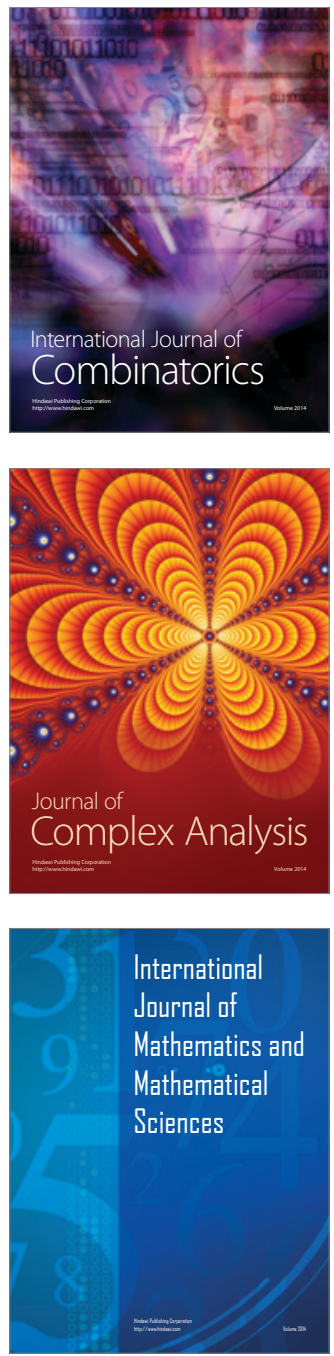
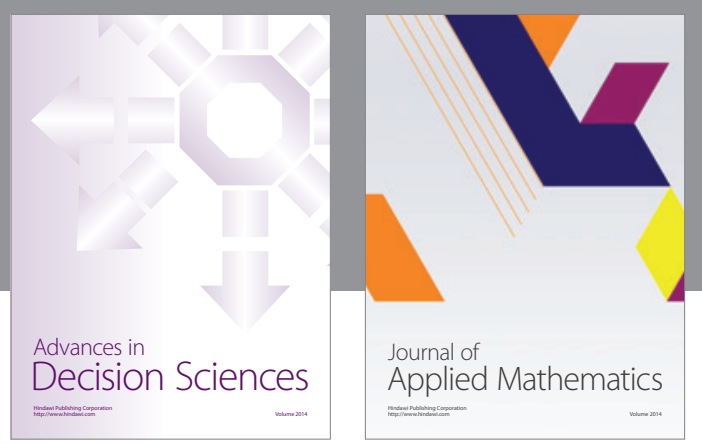

Algebra

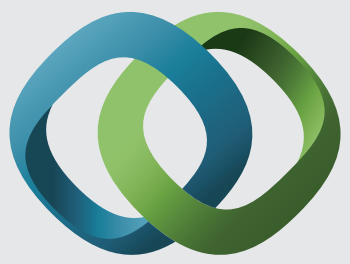

\section{Hindawi}

Submit your manuscripts at

https://www.hindawi.com
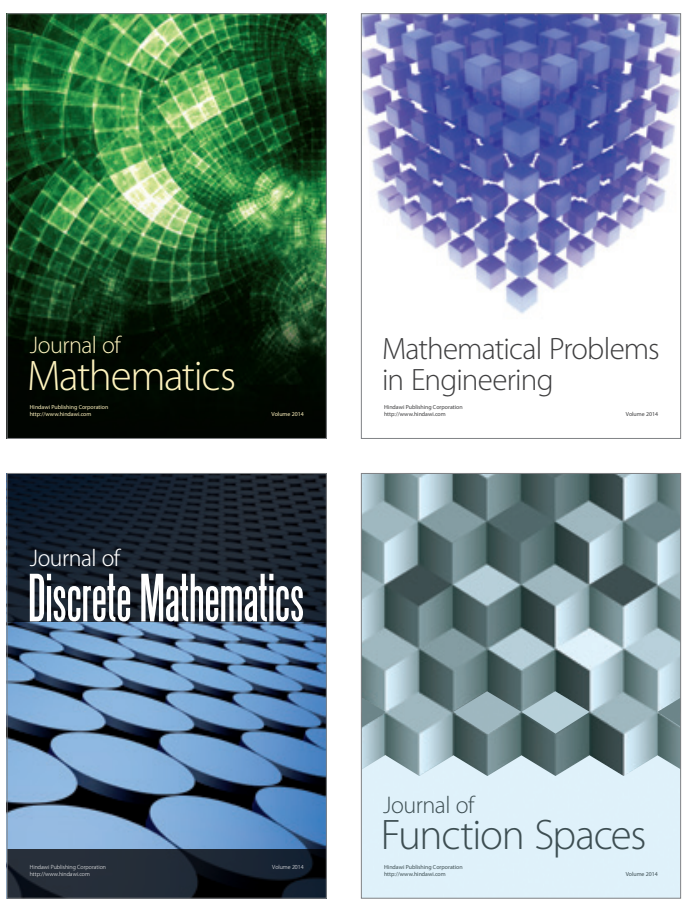

Mathematical Problems in Engineering
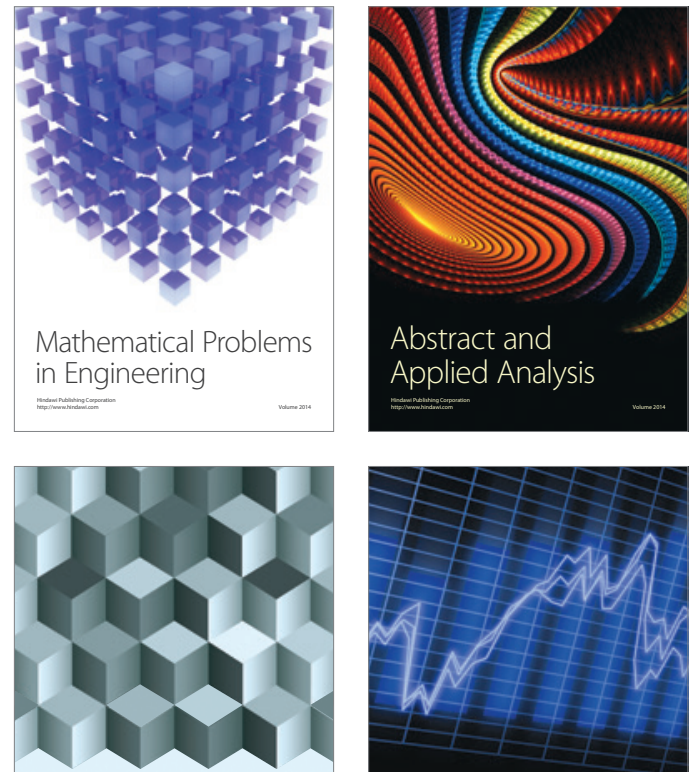

Journal of

Function Spaces

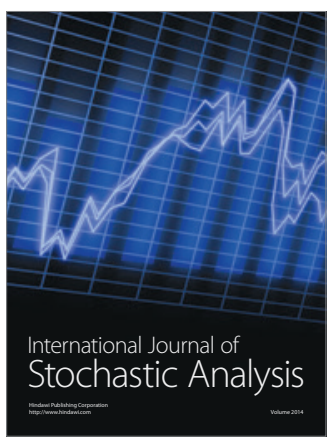

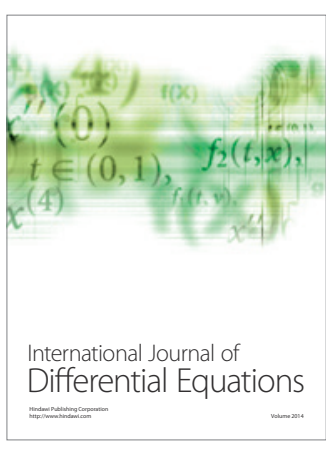
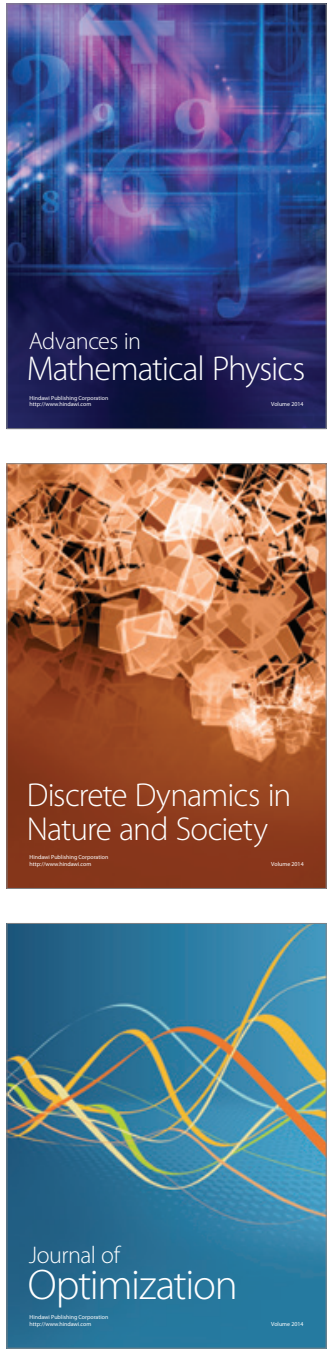